\title{
The VLT-FLAMES survey of massive stars: atmospheric parameters and rotational velocity distributions for B-type stars in the Magellanic Clouds ${ }^{\star}$ (Corrigendum)
}

\author{
I. Hunter ${ }^{1,2}$, D. J. Lennon ${ }^{2,3}$, P. L. Dufton ${ }^{1}$, C. Trundle ${ }^{1}$, S. Simón-Díaz ${ }^{4}$, S. J. Smartt ${ }^{1}$, R. S. I. Ryans ${ }^{1}$, and C. J. Evans ${ }^{5}$
}

${ }^{1}$ Astrophysics Research Centre, School of Mathematics \& Physics, The Queen's University of Belfast, Belfast, BT7 1NN, Northern Ireland, UK

e-mail: I.Hunter@qub.ac.uk

2 The Isaac Newton Group of Telescopes, Apartado de Correos 321, 38700, Santa Cruz de La Palma, Canary Islands, Spain

3 Instituto de Astrofísica de Canarias, 38200 La Laguna, Tenerife, Spain

${ }^{4}$ LUTH, Observatoire de Meudon, 5 place Jules Janssen, 92195 Meudon Cedex, France

5 UK Astronomy Technology Centre, Royal Observatory, Blackford Hill, Edinburgh, EH9 3HJ, UK

A\&A, 479, 541-555 (2008), DOI: 10.1051/0004-6361:20078511

Key words. stars: early-type - stars: atmospheres - stars: rotation - stars: evolution - galaxies: Magellanic Clouds - errata, addenda

An error occurred during the production process. Figure 1 was published twice. The corrected Figs. 1 and 2 are published below.

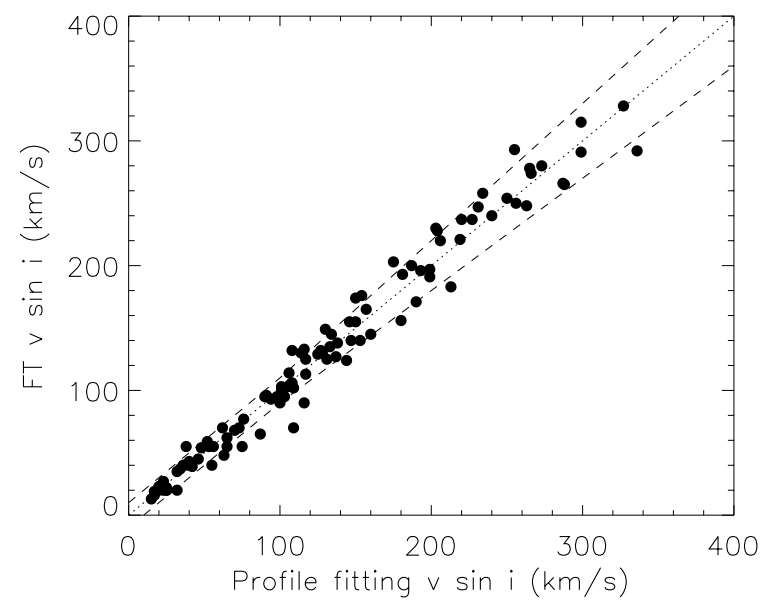

Fig. 1. Comparison of the projected rotational velocity derived by the profile fitting method and the Fourier transform method for the nonsupergiant objects in the sample. The dotted line indicates a one-to-one correlation. The dashed lines indicate a $10 \%$ or $10 \mathrm{~km} \mathrm{~s}^{-1}$ uncertainty, whichever is the larger.

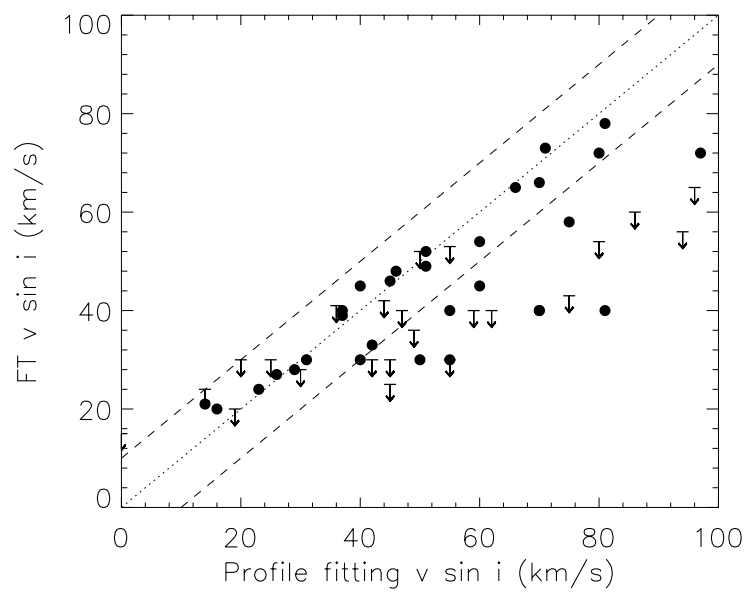

Fig. 2. Comparison of the projected rotational velocity $(v \sin i)$ derived by the profile fitting method and the Fourier transform method for the supergiant objects in the sample. The dotted line indicates a oneto-one correlation. The dashed lines indicate a $10 \mathrm{~km} \mathrm{~s}^{-1}$ uncertainty. Downward pointing arrows indicate those objects where it was only possible to derive upper limits to the $v \sin i$ from the Fourier method.

* Based on observations at the European Southern Observatory in programmes 171.0237 and 073.0234 . 